\title{
SPECTRAL FLUCTUATIONS OF INCOHERENT RADIATION AND MEASUREMENT OF LONGITUDINAL BUNCH PROFILE
}

\author{
M. S. Zolotorev \\ Center for Beam Physics, Accelerator and Fusion Research Division \\ Lawrence Berkeley National Laboratory, University of California, Berkeley, CA 94720 \\ and G. V. Stupakov \\ Stanford Linear Accelerator Center Stanford University, Stanford, CA 94309
}

\begin{abstract}
A method for measurement of ultrashort beam current profile $I_{b}(t)$ is proposed that is based on detecting fluctuations of the spectral intensity $P(\omega)$ of single bunch incoherent radiation. We show that the variance of the Fourier transform of the spectrum is proportional to the convolution function of the beam current. After the convolution function is found, using phase retrieval technique one can restore the shape of the pulse in many practical cases.
\end{abstract}

\section{INTRODUCTION}

Measurements of the longitudinal beam profile of a relativistic electron or positron beam is an important diagnostic tool in modern accelerators that is used both for routine monitoring and dedicated studies of beam physics. For bunch lengths in the range of picoseconds, such measurements can be performed by means of a streak camera. Shorter bunches usually require some kind of special techniques. Several methods have been proposed which have a potential of measuring ultrashort bunches. In Ref. [1], a coherent transition radiation emitted at wavelengths longer than or equal to the bunch length was used for analysis of subpicosecond electron pulses. In Ref. [2], Compton scattering of photons on interferometric pattern generated by two laser beams was proposed as a beam-profile monitor.

Recently, we have proposed a novel technique for measuring short bunches [3]. The method is based on the observation of the interferometric fringes produced by incoherent radiation of single bunch in a two beam interferometer. It was shown that fluctuations of the interferometric signal of a single bunch carry the information about the convolution function of the bunch current and allow to ascertain the bunch profile from analysis of the statistical properties of the fluctuations. In the present paper, we describe a new modification of the original idea that significantly simplifies the experimental apparatus and opens a possibility for designing a low-cost, easy-to-operate bunch length monitor.

\section{SETUP AND MEASUREMENT}

The schematic of the experimental setup of the measurement is shown in Fig. 1. Synchrotron light emitted by a bunch in a bend magnet passes through the spectrometer (S) and is measured by the CCD detector (D). The CCD selects a spectral range $\Delta \omega$ around the frequency $\omega_{0}$ in the

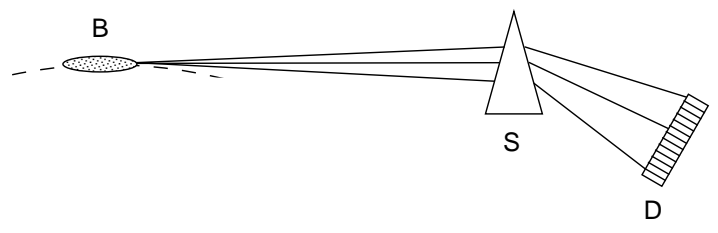

Figure 1: Schematic of the experimental setup. Synchrotron radiation of the beam (B) passes through the spectrometer (S) and is detected by the array of CCD (D).

interval $\left(\omega_{0}-\Delta \omega / 2, \omega_{0}+\Delta \omega / 2\right)$. Assuming that CCD has $N_{c h}$ channels, the frequency difference between the adjacent channels is $\Delta \omega / N_{c h}$. In order to be able to measure a bunch of length $\sigma_{b}$, the spectral resolution of the spectrometer should be better than $\tau_{b}^{-1}$, where $\tau_{b}=\sigma_{b} / c$. Also, the spectral width $\Delta \omega$ must be much larger than the inverse bunch length $\tau_{b}, \Delta \omega \gg \tau_{b}^{-1}$. Note that for the optimal performance the number of channels should be such that $\Delta \omega / N_{c h}$ is smaller the spectrometer resolution.

Let us denote the the detector signal in channel $m,(1<$ $\left.m<N_{c h}\right)$ as $P_{m}$. This signal is Fourier transformed,

$$
\Gamma_{k}=\sum_{m=1}^{N_{c h}} P_{m} e^{2 \pi i m k},
$$

and the quantity $\Gamma_{k}$ is stored. In case of a high repetition rate, one will have to use a fast shutter to prevent the exposure of the CCD by the light of the next bunch before the readout is completed. After accumulation of $N_{p}$ number of pulses, large enough for statistical analysis, the following quantity is computed

$$
d_{k}=\sum_{\alpha=1}^{N_{p}}\left|\Gamma_{k}^{(\alpha)}-\frac{1}{N_{p}} \sum_{\beta=1}^{N_{p}} \Gamma_{k}^{(\beta)}\right|^{2},
$$

where the superscripts $\alpha$ and $\beta$ indicate the pulse number. As we will show in the next section, the quantities $d_{k}$ give the convolution function of the particle density in the bunch (averaged over $N_{p}$ bunches), and, with some additional assumptions, allow to find the bunch profile.

\section{THEORY}

We assume that the emitted radiation can be described in terms of the classical field, which is true if the number of photons in the coherence volume is much greater than one. 
Let $E(t)$ be the electric field of the radiation of a single bunch. The spectrometer decomposes this field into Fourier harmonics and the detector measures the absolute value of the spectral intensity $P(\omega)$,

$$
P(\omega) \propto\left|\int_{-\infty}^{\infty} E(t) e^{i \omega t} d t\right|^{2}
$$

Due to the incoherent nature of the radiation, the result of each measurement fluctuates from channel to channel and from bunch to bunch. An example of a simulation of one measurement of the spectrum is shown in Fig. 2, (see details below).

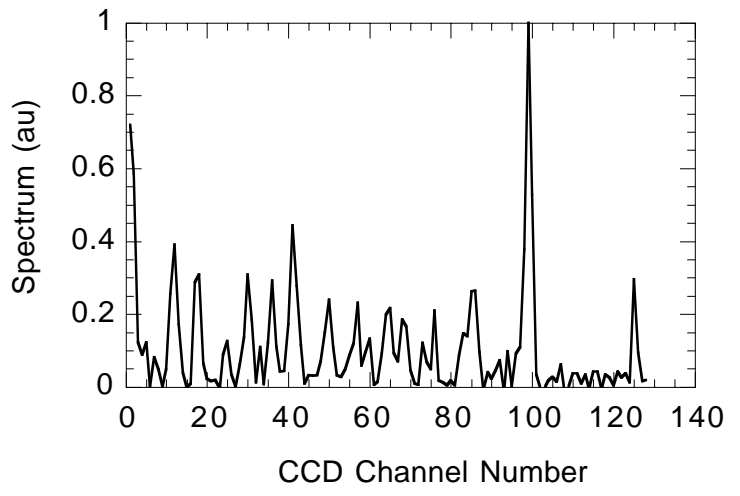

Figure 2: Simulation of a spectrum of a single bunch (in arbitrary units). The total number of channels $N_{c h}$ is 128 .

The quantity that carries information about the bunch profile is the Fourier transform of the measured spectrum which we denote by $\Gamma(\tau)$,

$$
\Gamma(\tau)=\int_{-\infty}^{\infty} P(\omega) e^{-i \omega \tau} d \omega
$$

In reality, this Fourier transform will be performed in discreet form as given by Eq. (1) after digitizing the signal from the CCD. Since $P(\omega)$ is a fluctuating function of the frequency, the function $\Gamma(\tau)$ will also exhibit random fluctuations.

To relate the statistics of the fluctuations of $\Gamma(\tau)$ to the pulse parameters, we represent the electric field of the pulse in the detector $E(t)$ as a product of two functions,

$$
E(t)=A(t) e(t),
$$

where $e(t)$ is a stationary complex-valued stochastic process [4], and $A(t)$ denotes a (deterministic) complex amplitude of the pulse, $A(t)=\sqrt{I(t)} e^{-i \varphi(t)}$, where $I(t)$ is the instantaneous radiation intensity and $\varphi(t)$ is the phase of the pulse envelope. The characteristic time of the variation of both functions $A(t)$ and $\varphi(t)$ is of the order of the pulse duration $\tau_{b}$. Related to the fluctuational part of the field $e(t)$ is a correlation function $K(\tau)$,

$$
K(\tau)=\left\langle e(t) e^{*}(t-\tau)\right\rangle
$$

where the angular brackets denote ensemble average. The function $K(\tau)$ oscillates with the frequency $\omega_{0}$ and falls off on the scale of the coherence time $\tau_{c o h} \approx \pi / \Delta \omega \ll \tau_{b}$ associated with the spectral width $\Delta \omega$. The function $\Gamma(\tau)$ is then equal,

$$
\Gamma(\tau)=\int_{-\infty}^{\infty} A(t) A^{*}(t-\tau) e(t) e^{*}(t-\tau) d t
$$

Using Eq. (6), we can easily find the average value of $\Gamma(\tau)$,

$$
\begin{aligned}
& \langle\Gamma(\tau)\rangle=\mathrm{K}(\tau) \int_{-\infty}^{\infty} A(t) A^{*}(t-\tau) d t \\
& \approx \mathrm{K}(\tau) \int_{-\infty}^{\infty} I(t) d t .
\end{aligned}
$$

The quantity $\langle\Gamma(t)\rangle$ is proportional to the total charge of the bunch and does not contain information about the bunch profile.

Now, we want to show that the fluctuations of $\Gamma(\tau)$ around its average value indeed carry the information about $I(t)$. To this end, we calculate the absolute value of the variance $d_{\Gamma}(\tau)$ of the fluctuations of $\Gamma(t)$. For $d_{\Gamma}(\tau)$ we have

$$
\begin{aligned}
& d_{\Gamma}(\tau)=\left\langle|\Gamma(\tau)|^{2}\right\rangle-|\langle\Gamma(\tau)\rangle|^{2} \\
= & \int_{-\infty}^{\infty} \int_{-\infty}^{\infty} d t d t^{\prime} A(t) A^{*}(t-\tau) A^{*}\left(t^{\prime}\right) A\left(t^{\prime}-\tau\right) \\
\times & \left\langle e(t) e^{*}(t-\tau) e^{*}\left(t^{\prime}\right) e\left(t^{\prime}-\tau\right)\right\rangle-|K(\tau)|^{2} \\
\times & \int_{-\infty}^{\infty} \int_{-\infty}^{\infty} d t d t^{\prime} A(t) A^{*}(t-\tau) A^{*}\left(t^{\prime}\right) A\left(t^{\prime}-\tau\right) .
\end{aligned}
$$

For a normal stochastic process, the fourth order correlators are reduced to the sum of the products of the second order correlators, yielding

$$
\begin{array}{r}
\left\langle e(t) e^{*}(t-\tau) e^{*}\left(t^{\prime}\right) e\left(t^{\prime}-\tau\right)\right\rangle \\
=\left\langle e(t) e^{*}(t-\tau)\right\rangle\left\langle e^{*}\left(t^{\prime}\right) e\left(t^{\prime}-\tau\right)\right\rangle \\
+\left\langle e(t) e^{*}\left(t^{\prime}\right)\right\rangle\left\langle e^{*}(t-\tau) e\left(t^{\prime}-\tau\right)\right\rangle \\
=|K(\tau)|^{2}+\left|K\left(t-t^{\prime}\right)\right|^{2} .
\end{array}
$$

The first term on the right hand side of Eq. (9) appears in the theory of Hunbury Brown - Twiss interferometry [6], however, it does not contain information about the pulse shape. It will be canceled by the last term on the right hand side of Eq. (9). From Eqs. (9) and (9) on can find

$$
d_{\Gamma}(\tau)=\int_{-\infty}^{\infty}|K(\xi)|^{2} d \xi \times \int_{-\infty}^{\infty} d t I(t) I(t-\tau)
$$

Note that $d_{\Gamma}(\tau)$ is proportional to the convolution of the intensity $I(t)$. For the discrete Fourier transformation, 
$d_{k}$ given by Eq. (2) represent the function $d_{\Gamma}(\tau), d_{k}=$ $d_{\Gamma}(k / \delta \omega)$.

Strictly speaking, knowledge of the convolution function does not allow a unique restoration of $I(t)$. However, as was shown in Ref. [7], using a phase retrieval technique allows to restore the beam profile in many practical cases.

The above consideration assumed that the quantum fluctuations of the number of photons in the detector are negligible. We can easily derive the condition for the beam intensity when this is requirement is met. The number of synchrotron photons, $n_{p h}$ that reach the detector (they are radiated from $(1 / \gamma)\left(\omega_{c} / \omega_{0}\right)^{1 / 3}$ radians of the beam trajectory, where $\omega_{c}$ is the critical frequency for the synchrotron radiation) is equal [5]

$$
\left\langle n_{p h}\right\rangle \approx \alpha N_{e} \delta \omega / \omega_{0}
$$

where $\alpha$ is the fine structure constant, $N_{e}$ is the number of electrons in the bunch, and $\delta \omega$ is the spectral width per one channel, $\delta \omega=\Delta \omega / N_{c h}$. The condition $\left\langle n_{p h}\right\rangle \gg 1$ gives a limitation on $N_{e}$ from below. Note, that in this estimate we assumed that the beam radiation is coherent in the transverse direction, otherwise an additional factor would appear in Eq. (11).

If condition $\left\langle n_{p h}\right\rangle \gg 1$ is not met, quantum fluctuations superimpose on the random fluctuations described above. However, the information about the pulse shape is still present in the measured signal, although a larger statistics would be needed to suppress the additional noise introduced by the quantum fluctuations.

\section{COMPUTER SIMULATIONS}

We have performed computer simulation of the measurement of fluctuating spectra. The code simulates incoherent radiation of a large number $N_{e}$ of independent sources (electrons) that are distributed according Gaussian function with the rms value $\tau_{b}$. The average intensity of incoherent radiation is given by $I(t)=I_{0} \exp \left(-t^{2} / 2 \tau_{p}^{2}\right)$.

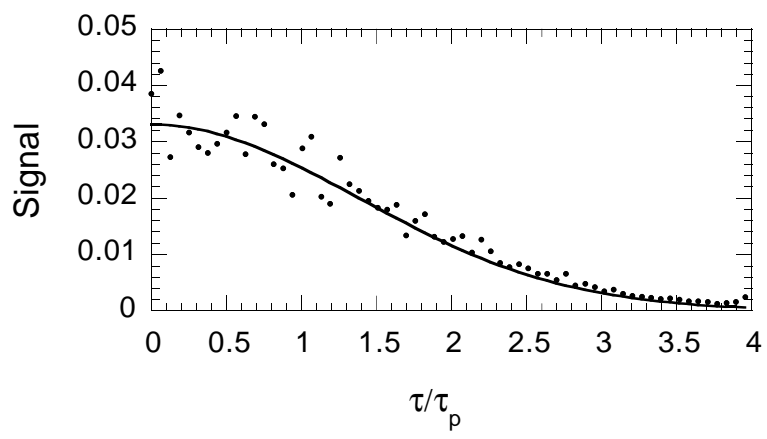

Figure 3: Simulation of a measurement averaged over 100 bunches. Dots represent simulated values of $d_{k}$, and the curve is a theoretical convolution function for the beam density.

The parameters for this particular example are: $\tau_{b}=$ $17 \mathrm{ps}$ (rms bunch length $\sigma_{b}=0.5 \mathrm{~cm}$ ), the wavelength of the central frequency $\lambda=2 \pi c / \omega_{0}=6300 \AA$. We assume a standard spectrometer with dispersion of about $10 \AA / \mathrm{mm}$ and a CCD with 128 channels with a pixel size of $10 \mu \mathrm{m}$. A spectrum of a single bunch is shown in Fig. 2, and the result of the simulation of the measurement of $d_{\Gamma}(\tau)$ using averaging over 100 pulses is shown in Fig. 3 with a solid line showing theoretical prediction for a Gaussian beam, $d_{\Gamma}^{(t h)}(\tau)=$ const $\exp \left(-t^{2} / 4 \tau_{b}^{2}\right)$. A Gaussian fit to the measurement gives for $d_{\Gamma}(\tau)$ the rms width of 1.38 (instead of the theoretical value of $\sqrt{2}$ ) which translates into the measurement error of about $2 \%$.

Note that Eq. (11) gives for the parameters cited above the following limitation for the number of particles in the bunch, $N_{e} \gg 5 \times 10^{7}$.

\section{CONCLUSION}

In summary, a technique is proposed, capable of measuring short pulses of incoherent radiation. It is based on the observation and statistical analysis of the bunch spectrum fluctuations. Although we assumed synchrotron radiation above, the nature of the radiation is not important, and, for example, transition radiation can be used as well. Typically, averaging over fluctuations requires accumulation of many (on the order of $10^{2}$ ) pulses for a singe measurement, however, a modification of the method is possible in which one can perform averaging over different spectral intervals in a single pulse.

An important feature of the method is that it can be used for bunches with the lengths ranging from a centimeter to tens of microns.

Experimental verification of the method is under way at the synchrotron source ALS at the Lawrence Berkeley Laboratory.

\section{ACKNOWLEDGMENTS}

The authors are thankful to W. Leemans, K.-J. Kim and A. Zholents for useful discussions. This work was supported by Department of Energy contracts DE-AC03-76SF00515 and DE-AC03-76SF00098.

\section{REFERENCES}

[1] P. Kung et al., Phys. Rev. Lett., 73, 967 (1994).

[2] T. Shintake. KEK-PREPRINT-96-81, Jul 1996. 20pp. Invited talk at 7th Beam Instrumentation Workshop (BIW 96), Argonne, IL, 6-9 May 1996.

[3] M.S. Zolotorev and G.V. Stupakov. SLAC Preprint SLACPUB-7132. March, 1996.

[4] D. R. Cox and H. D. Miller, The Theory of Stochastic Processes, (John Wiley Inc., New-York, 1965).

[5] M. Sands. Preprint SLAC-121, p.117, (1970).

[6] W. Lauterborn, T. Kurz and M. Wiesenfeldt. Coherent Optics, (Springer, 1995).

[7] R. Lai, A.J. Sievers. Micro Bunches Workshop, AIP Conference Proceedings 367 (NY), p. 312 (1995). 